\title{
Note
}

\section{Errors in the Numerical Integration of a Linearized Equation for Particle Flows}

\section{Particle Flow Modeling}

Particle laden and gas-droplet flows are common in a wide range of practical problems. Anyone who has driven a car through a bug infested region is familiar with the multiphase dynamics of insects in air. Large bugs impact, while small bugs slip over the roof. The difference in their fate is due to their differing response to changes in their slip velocity (slip velocity is the bug's velocity relative to the air flow).

When particles, droplets or bubbles are transported in an unsteady velocity field, determining the particle motion analytically is exceedingly difficult and numerical solutions are employed. In typical engineering problems the particle motion is a desired solution.

Examples include dust ingestion in turbomachinery $[1,2]$, solid propellant motion in rocket nozzles [3], raindrop impingement on aircraft surfaces [4], and pulverized coal combustion [5]. In each of these examples and in many others, the particles governing differential equations (namely Newton's second law) are integrated in time over small steps. In the above analyses the drag force which is a function of slip velocity squared has been linearized.

This linearization introduces errors, except in the case of a particle in Stoke's flow (since here the drag force is a linear function of slip velocity). Most authors have inadverdently introduced errors in their particle integration by using the linearization. Now we will show how errors in the linearized equations may greatly exceed the global error of integration. A simple analysis indicates a new criterion for choosing the time step. In this way the economy of integrating a linear equation can be fully exploited.

\section{The Governing Equations}

As shown by Taylor [6] the motion of a dilute suspension of droplets or particles of density $\rho$ moving with the velocity components $u$ and $v$ in a fluid of density $\rho_{f}$ flowing with the velocity components $u_{f}$ and $v_{f}$ can be approximated by:

$$
\frac{d \mathbf{u}}{d t}=\frac{\left(\mathbf{u}_{f}-\mathbf{u}\right)}{\tau} g
$$


where

$$
\tau=\frac{2}{9} \frac{\rho \sigma^{2}}{\mu_{f}}
$$

is constant for a given particle radius, $\sigma$, while

$$
g=\frac{C_{D} q_{s}(2 \sigma)}{24 v_{f}}
$$

is a function of the slip speed,

$$
q_{s}=\left[\left(u_{f}-u\right)^{2}+\left(v_{f}-v\right)^{2}\right]^{1 / 2} .
$$

These equations were first derived by Taylor in his work with raindrop impingement on aircraft wings. We have neglected lift, gravity, pressure, and Basset, Magnus and Brownian motion forces, since the vast majority of two phase flows are drag dominated.

Clearly if $g$ is considered a constant then the equation is linear. Physically this corresponds to assuming the particle's Reynolds number is time invariant over an integration step. Thus in the approximate form $g$ is specified by the initial conditions. In his original analytical calculations Taylor assumed $g=1$, corresponding to the ideal Stoke's flow.

The equations are non-dimensionalized as follows:

$$
\begin{gathered}
T=t / \tau, \quad U=u / U_{\infty}, \quad V=v / U_{\infty}, \\
Q_{s}=q_{s} / U_{\infty} .
\end{gathered}
$$

Yielding from (1):

$$
\frac{d \mathbf{U}}{d T}=-\left(\mathbf{U}-\mathbf{U}_{f}\right) Q_{s} \frac{C_{D} R_{\infty}}{24}
$$

where $R_{\infty}=\left(2 \sigma U_{\infty} / v_{f}\right)$ is a fluid flow Reynolds number based on particle diameter and $U_{\infty}$ so it is constant for a given particle size and up-stream flow. Again, in the linear approximation $Q_{s}$ is held constant, while in Taylor's original formulation $Q_{s}$ is a function of the dependent variable.

\section{Absolute Error AsSessment}

The non-dimensional force magnitude in a two-dimensional flow is given by

$$
|F|=\sqrt{\left(F_{x}\right)^{2}+\left(F_{y}\right)^{2}}
$$


where

$$
F_{x}=\frac{24}{R_{\infty} C_{D}} \frac{d U}{d T} \quad \text { and } \quad F_{y}=\frac{24}{R_{\infty} C_{D}} \frac{d V}{d T} .
$$

The absolute error $E$ (i.e., Taylor force-Linearized force) per unit time step $\Delta T$ is found by substituting the right-hand side of (2) into (3):

$$
\begin{aligned}
E= & \left\{\left[\left(U-U_{f}\right) Q_{s}\right]^{2}+\left[\left(V-V_{f}\right) Q_{s}\right]^{2}\right\}^{1 / 2} \\
& -\left\{\left[\left(U-U_{f}\right) \widetilde{Q}_{s}\right]^{2}+\left[\left(V-V_{f}\right) \widetilde{Q}_{s}\right]^{2}\right\}^{1 / 2} / \Delta T ;
\end{aligned}
$$

all variables are evaluated at the end of the time step where $T=T_{0}+\Delta T$, except $\Phi_{s}$, which is evaluated by the initial conditions as $\tilde{Q}_{s}=Q_{s}\left(T_{0}\right)$. After some manipulation the error becomes

$$
E=Q_{s}\left(T_{0}+\Delta T\right) \frac{Q_{s}\left(T_{0}+\Delta T\right)-Q_{s}\left(T_{0}\right)}{\Delta T} .
$$

Note that in the limit of vanishingly small $\Delta T$ this expression is zero, implying the obvious result that the Taylor force and the linearized force agree initially.

Now expand the slip speed $Q_{s}$ in a Taylor series (neglecting second order terms) and substitute for $Q_{s}\left(T_{0}+\Delta T\right)$ in (4), yielding

$$
\begin{aligned}
E & =\left[Q_{s}\left(T_{0}\right)+\dot{Q}_{s}\left(T_{0}\right) \Delta T\right] \dot{Q}_{s}\left(T_{0}\right)+O(\Delta T)^{2} \\
& =\frac{1}{2} \frac{d Q_{s}^{2}}{d T}+\left[\dot{Q}_{s}\left(T_{0}\right)\right]^{2} \Delta T+O(\Delta T)^{2}
\end{aligned}
$$

This result can be interpreted as equating the absolute error per unit time step to the sum of the rate of change of the slip speed kinetic energy plus the slip acceleration squared multiplied by the time step. Physically such a situation is found at a stagnation point. The gas or liquid is decelerated to zero speed, however the higher inertia particles continue to move, giving rise to an increasing slip speed. In other examples, regions of high fluid shear can produce sudden changes in the particles slip speed. For flow towards a stagnation point the error is always positive.

\section{Relative Error Estimates}

The interpretation of (5) is of use in determining the source of errors, but an estimate of error as a function of time step, $\Delta T$, is of greater pragmatic value. The Taylor equation (1) and linearized form $\left(Q_{s}=\right.$ constant) are exactly integrable for the one dimensional flow of a fluid whose velocity varies slowly over a time step.

In Taylor's form we have,

$$
\int_{T_{0}}^{T_{0}+\Delta T} \frac{d U}{\left(U-U_{f}\right)^{2}}=-\frac{C_{D} R_{\infty}}{24} \int_{T_{0}}^{T_{0}+\Delta T} d T .
$$


For the linearized case

$$
\int_{T_{0}}^{T_{0}+\Delta T} \frac{d U}{U-U_{f}}=-\bar{Q}_{s} \frac{C_{D} R_{\infty}}{24} \int_{T_{0}}^{T_{0}+\Delta T} d T .
$$

We have assumed that the drag coefficient is a weak function of particle speed over one time step.

Equations (6) and (7) yield, respectively,

$$
\begin{aligned}
& Q_{s}\left(T_{0}+\Delta T\right)=\left[\frac{1}{Q_{s}\left(T_{0}\right)}+\frac{C_{D} R_{\infty} \Delta T}{24}\right]^{-1}, \\
& Q_{s}\left(T_{0}+\Delta T\right)=\exp \left[\ln Q_{s}\left(T_{0}\right)-\widetilde{Q}_{s} \frac{C_{D} R_{\infty}}{24} \Delta T\right],
\end{aligned}
$$

where $Q_{s}\left(T_{0}\right) \equiv \tilde{Q}_{s}$ by definition.

In Fig. 1 the relative error in the integrated slip speed (from Eqs. (8) and (9)) is plotted for various initial slip speeds $Q_{s}\left(T_{0}\right)$ as a function of the reduced time step $\left(\Delta T C_{D} R_{\infty} / 24\right)$. Typical industrial flows have reduced time steps varying between 0.01 and 10.0. Particles traveling nearly with the fluid (i.e., small slip speed) have less relative error than particles with a high initial slip speed. This is because Eq. (5) predicts the linearized force to underestimate the Taylor force. From Eq. (1) we see the force increases with slip speed, hence the underestimation increases with initial slip speed.

The enlarged detail in Fig. 1 indicates that the slip speed error can be made less than $1 \%$ if the reduced time step is less than 0.15 . Furthermore the slope of the

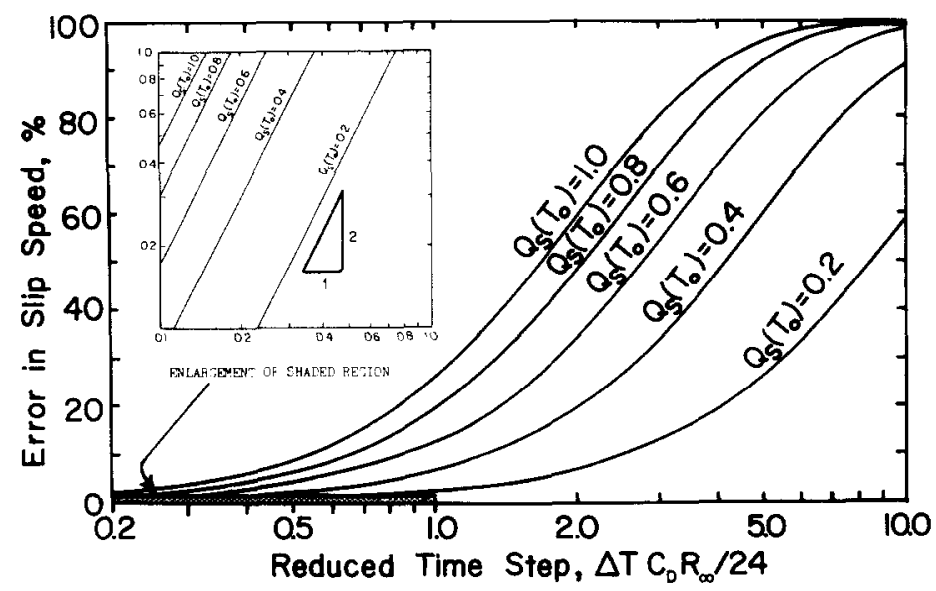

FIG. 1. Analytically determined errors in the particle's slip speeds as a function of the reduced time step of integration. The small inset figure has the same axes as the large figure, namely the percentage relative error versus the reduced time step. The error is always less than $1 \%$ if the reduced time step is less than 0.15 . 
curves in this magnified $\log -\log$ is approximately 2.0 . Thus the relative slip-speed error is proportional to the reduced time step squared.

A typical particle integration routine such as a fourth order Runge-Kutta scheme has errors proportional to the time step raised to the fourth power. Hence errors in the linearized equations can exceed the global error of integration.

However, in fluid regions of low shear the particle slip speeds are low and the linearization errors are small. This suggests a simple numerical method that can be incorporated in an integration routine with variable step size control. A local check of the reduced time step can be computed periodically and Eqs. (8) and (9) employed to determine an accurate upper bound on $\Delta T$. In this way an algorithm with variable step size could efficiently exploit the linearized equations.

\section{REFERENCES}

1. M. F. Hussein and W. Tabakoff, J. Aircraft $10(1973), 434$.

2. G. P. TILly, Wear 14 (1969), 63.

3. J. H. NeIlson ANd A. Gilchrist, J. Fluid Mech. 33 (1968), 131.

4. R. J. BRUN et al., "Impingement of Cloud Droplets on Aerodynamic Bodies as Affected by Compressibility of Air Flow around the Body," NACA Tech. Note No. 2903 (1953).

5. C. T. Crowe ANd D. E. Stock, "Computer Solution of Two Dimensional Fluid-Particle Flows," presented at the International Symposium on Finite Element Methods in Flow Problems, Swansea, Wales, Jan. 7-11 (1974).

6. G. I. TAylor, Aero. Res. Com., Rep. and Mem. No. 2024 (1940).

RECEIVED: May 13, 1981

J. A. LAITONE

University of Michigan Department of Mechanical Engineering and Applied Mechanics, Ann Arbor, Michigan 48109

E. V. LAITONE

University of California Department of Mechanical Engineering Berkeley, California 94720 